\title{
REFERENCIA AL ARTÍCULO "USO DE LOS ANÁLISIS ESTADÍSTICOS EN LOS ARTÍCULOS ORIGINALES DE ACTAS UROLÓGICAS ESPAÑOLAS. ACCESIBILIDAD PARA EL LECTOR"
}

\author{
F. MILLÁN RODRÍGUEZ
}

Servicio de Urología. Fundació Puigvert. Barcelona.

Actas Urol Esp. 27 (7): 566, 2003

Señor director:

En primer lugar, quisiera resaltar el interés del original "Uso de los análisis estadísticos en los artículos originales de Actas Urológicas Españolas. Accesibilidad para el lector", publicado en la Revista Actas Urológicas Españolas 27 (2): 103-109, 2003, porque con este estudio de los análisis estadísticos de los originales publicados en nuestra revista, se ilustra la necesidad que tenemos los urólogos españoles de ir adquiriendo progresivamente los conocimientos adecuados para poder realizar una lectura crítica de la literatura científica. No obstante, aún compartiendo bastantes elementos de este trabajo, considero que la evaluación de la calidad de un artículo científico no debe reducirse únicamente al análisis del test estadístico utilizado (que ha sido el criterio empleado en este estudio) y mucho menos basarse en la clasificación jerárquica de la Tabla I, que considero arbitraria. Así, por ejemplo, sostener que una prueba no paramétrica (por ejemplo $U$ de Mann-Whitney) tiene más valor (criterio 4) que la prueba t de Student (criterio 2) lo considero incorrecto, entre otras cosas, porque es menos potente y no proporciona los intervalos de confianza. De este modo, si se cumplen las condiciones de aplicación, siempre es mejor usar la t de Student que la $U$ de Mann-Whitney, por lo que no entiendo que en la última conclusión de este original se sorprendan de no haber encontrado ningún trabajo que use tests no paramétricos. Otro ejemplo ilustrativo de la arbitrariedad de esta clasificación podría ser el siguiente. Un ensayo clínico multicéntrico aleatorizado doble ciego bien diseñado y con una buena validez interna podría usar como test estadístico la $\mathrm{t}$ de Student (criterio 2) para comparar por ejemplo el flujo máximo medio pre y postratamiento en la evaluación de un fármaco para el tratamiento de la
HBP. En cambio, un estudio retrospectivo de factores pronósticos, por ejemplo, de recurrencia de tumor vesical podría realizarse mediante un análisis de supervivencia de Kaplan-Meier (criterio 16) y sin embargo el estudio del ensayo clínico en HBP presenta un nivel de evidencia científica mayor que la revisión retrospectiva de tumor vesical. Por todo ello, considero conveniente recordar que la calidad de un trabajo no depende únicamente del test estadístico utilizado (y mucho menos evaluado según esta clasificación), sino de si está diseñado de forma correcta para poder evaluar la hipótesis del objetivo que se quiere demostrar. Y esto no depende de la complejidad del aprendizaje del cálculo del test estadístico usado sino de la respuesta a preguntas tan sencillas como por ejemplo en el caso de un ensayo clínico: ¿Es interesante el objetivo planteado? ¿Los tratamientos se asignaron aleatoriamente? ¿La aleatorización fue ciega? ¿Acabaron el estudio todos los pacientes? ¿El resultado final es clínicamente relevante? ¿Este resultado va a ser útil para mis pacientes? De este modo, si no centramos el análisis de un artículo únicamente en la parte matemática de los tests estadísticos (sin lugar a dudas, siempre necesaria) sino que lo abrimos también a consideraciones clínicas como las preguntas que se acaban de exponer contribuiremos a hacer más accesible e inteligible la metodología científica al urólogo clínico.

Respetuosamente.

Dr. F. Millán Rodríguez

Servicio de Urología. Fundació Puigvert

C/ Cartagena, 340

08025 Barcelona

(Trabajo recibido el 21 abril de 2003) 